Referencia para citar este artículo: Díaz-Barriga Arceo, F., Vázquez-Negrete, V. I., \& Díaz-David, A. (2019). Sentido de la experiencia escolar en estudiantes de secundaria en situación de vulnerabilidad. Revista Latinoamericana de Ciencias Sociales, Niñez y Juventud, 17(1), 237-252. doi: https://dx.doi.org/10.11600/1692715x.17114

\title{
Sentido de la experiencia escolar en estudiantes de secundaria en situación de vulnerabilidad*
}

\author{
FRIDA DÍAZ-BARRIGA ARCEO** \\ Profesora Universidad Nacional Autónoma de México, México.
}

VERÓNICA ISABEL VAZQUEZ-NEGRETE
Integrante Grupo de Investigación en Docencia, Diseño Educativo y TIC de la Universidad Nacional Autónoma de México, México.

AdolFo DíaZ-DAVID ${ }^{* * * *}$

Integrante Grupo de Investigación en Docencia, Diseño Educativo y TIC de la Universidad Nacional Autónoma de México, México.

\section{Artículo recibido en enero 15 de 2018; artículo aceptado en marzo 8 de 2018 (Eds.)}

- Resumen (analítico): se reporta una investigación cualitativa biográfico-narrativa, que recupera la voz de los y las estudiantes de secundaria respecto a la manera en que viven la institución escolar. Con el propósito de explorar la identidad narrativa autobiográfica de adolescentes en situación de vulnerabilidad académica y social, se solicitó a un grupo de 33 estudiantes mexicanos de tercer grado de secundaria en riesgo de abandono y rezago escolar, que generaran y digitalizaran un relato digital sobre sus vivencias escolares. Se buscó comprender los factores asociados al desvanecimiento progresivo del sentido de la educación y del aprendizaje escolar. Las narrativas de los estudiantes desvelaron la vida socioemocional de los adolescentes, la crítica a la normativa escolar y a la autoridad, la aceptación entre pares, el acoso escolar, las carencias en las instalaciones y servicios educativos.

Palabras clave: adolescentes, estudiantes de bajo rendimiento, identidad, narrativa (Tesauro Iresie).

\section{Meanings of the high school experience for students in vulnerable situations}

- Abstract (analytical): This study used a qualitative biographical-narrative approach to capture students' voices regarding the way they experience secondary school. In order to explore the autobiographical narrative identity of adolescents in a situation of academic and social vulnerability,

Este artículo de investigación científica y tecnológica es resultado de una investigación que se realizó entre enero y junio de 2017 como parte del proyecto «Aprender con sentido. Estrategias, instrumentos y prácticas de personalización del aprendizaje escolar» dirigido por César Coll (Universidad de Barcelona), apoyado por el Instituto de Tecnologías para el Aprendizaje (Intea) y la Fundación SM de España y México. Se reportan avances de la sede México, coordinada por la primera autora del artículo. Número de código del proyecto de investigación otorgado por la instancia financiadora, Dirección General de Asuntos del Personal Académico (Dgapa) a través del Programa de Apoyo a Proyectos para la Innovación y Mejoramiento de la Enseñanza: Papime PE300217. Área: Ciencias de la Educación; subárea: Educación General.

** Doctora en Pedagogía, profesora titular de la Facultad del Psicología (Unam) e integrante del Sistema Nacional de Investigadores, nivel 3. Coordina el Grupo de Investigación en Docencia, Diseño Educativo y TIC (Giddet). Orcid: 0000-0001-8720-1857. Índice H5: 29. Correo electrónico: diazfrida@prodigy.net.mx

*** Licenciada en Psicología y egresada de la Maestría en Pedagogía (Facultad de Filosofía y Letras, Unam). Tutora en la escuela secundaria donde se realizó esta investigación, en la cual formó parte del grupo de investigación como becaria de la Fundación SM México. Orcid: 0000-00020910-4215. Índice H5: 1. Correo electrónico: veronicaisabel06@gmail.com

**** Licenciado en Psicología (Unam) egresado del área de Neurociencias y con formación en tecnologías de la información y comunicación. Participó en esta investigación cubriendo la función de co-tutor de los estudiantes y como asesor en el proceso de digitalización de los relatos. Orcid: 0000-0003-0968-654X. Correo electrónico: fito.dd@hotmail.com 
the researchers selected a group of 33 Mexican students who were at risk of dropping out and falling behind and asked them to create a digital story about their school experiences. We sought to understand the factors associated with the progressive reduction in the meaning of education and school learning for these students. Their narratives highlighted the importance of their socio-emotional lives, criticism of school regulations and authority, the relevance of friendship and acceptance among peers, the way they conceive bullying and the lack of educational facilities and services in affecting their view of education.

Key words: adolescents, underperforming students, identity, narrative (Iresie Thesaurus).

\section{Senso de experiência escolar em estudantes do ensino médio em situação de vulnerabilidade}

- Resumo (analítico): uma pesquisa qualitativa biográfica-narrativa é relatada, que recupera a voz dos alunos sobre a forma como vivem em suas instituições escolares. Para explorar a identidade narrativa autobiográfica de adolescentes em situação de vulnerabilidade acadêmica e social, um grupo de 33 estudantes mexicanos de ensino médio em risco de abandonar ou atrasar os estudos produziram e digitalizaram uma história sobre suas experiências escolares. Buscamos compreender os fatores associados ao desvanecimento progressivo do senso de educação e aprendizagem escolar. As narrativas dos alunos revelaram a vida social dos adolescentes, a crítica dos regulamentos $e$ autoridades escolares, amizade e aceitação entre pares, bullying, deficiências nas instalações e serviços educacionais.

Palavras-chave: adolescentes, baixo desempenho escolar, identidade, narrativa.

\section{-1. Introducción. -2. Adolescentes en situación de vulnerabilidad. -3. Identidad narrativa y fondos de identidad. -4. Metodología. -5. Resultados. -6. Conclusiones. -7. Lista de referencias.}

\section{Introducción}

En este trabajo nos acercamos a recuperar la voz de un grupo de adolescentes, mujeres y varones, estudiantes mexicanos de secundaria pública, que se encontraban en situación de vulnerabilidad académica y social, en la medida en que mostraban una historia de reprobación escolar, o bien habían sido reportados y sancionados reiteradamente por sus profesores y directivos debido a problemas de comportamiento. Estos adolescentes cursaban tercer grado de secundaria y tenían varias materias reprobadas, por lo que se encontraban en situación de rezago escolar y en riesgo de expulsión o abandono de la institución educativa. Por tal motivo, el subdirector conformó un grupo «especial», a fin de que los y las adolescentes en cuestión recibieran apoyo psicopedagógico específico en el espacio curricular de Tutoría, con la meta de remontar la reprobación, aprender estrategias de estudio efectivas y aumentar su motivación o disposición por aprender. Esto dio la pauta a un proceso de investigación e intervención educativa a cargo de los autores del presente artículo, quienes realizaron un estudio cualitativo de los fondos de identidad del aprendiz (Esteban-Guitart, 2016) a través de narrativa digital autobiográfica (Lambert, 2010), con el propósito de desarrollar una serie de dispositivos pedagógicos efectivos (Sanjurjo, 2009) que permitieran desvelar y promover el sentido en el aprendizaje escolar. En este artículo se reporta lo concerniente a la elaboración de las narrativas elaboradas por los y las adolescentes en el formato de relatos digitales personales y se analiza lo concerniente a los fondos de identidad allí plasmados.

De acuerdo con Coll $(2009,2016)$, un fenómeno contemporáneo presente en todos los niveles del sistema educativo y en numerosos países tanto desarrollados como en vías de serlo, consiste en la pérdida de sentido que los estudiantes manifiestan hacia la institución escolar y hacia el aprendizaje formal. Muestra de ello son los bajos resultados educativos, los diversos estudios que encuentran la baja motivación por el aprendizaje académico, la problemática en la convivencia y bienestar en las escuelas. Al mismo tiempo, el cuestionamiento al sentido de la escuela está presente en los continuos 
debates respecto a si ésta transmite un patrimonio cultural relevante y prepara para el futuro a los jóvenes, dado el nuevo entorno social y económico, marcado por la incertidumbre, la inequidad y la irrupción de las tecnologías digitales.

El sentido del aprendizaje se relaciona con la disposición e interés por aprender de parte de los estudiantes, en la medida en que los aprendizajes se puedan vincular con situaciones que resultan motivantes y relevantes para su vida, mientras respondan a sus necesidades e intereses y promuevan en ellos una imagen positiva de sus capacidades para aprender. En muchos contextos educativos la pérdida de sentido se relaciona con el capital cultural y social de los estudiantes, aunado a la falta de pertinencia del currículo escolar y de las experiencias educativas en las aulas, desconectadas de su experiencia de vida, sus intereses y de prácticas socioculturales que consideran importantes en su grupo etáreo y comunidad ${ }^{1}$. Diversos investigadores han encontrado que la educación secundaria resulta poco significativa y pertinente para los adolescentes mexicanos, debido a que en su conformación, prácticas y formas de relación pedagógica se excluye la realidad, diversidad cultural, intereses e identidad de los mismos (Arreola, 2017; Reyes, 2016). En los dos estudios antes referidos, mediante entrevistas semiestructuradas y observaciones etnográficas en las escuelas, se logró documentar, desde la perspectiva de los estudiantes, un desinterés por el contenido escolar, las tareas a realizar y las actividades de enseñanza-aprendizaje, porque no les veían utilidad ni aplicación en la vida real presente ni futura, además de que los docentes daban clases «aburridas», eran «autoritarios», o no los tomaban en cuenta.

En este trabajo postulamos que para poder entender la construcción del sentido en el educando en torno a su experiencia escolar y apoyar una mayor disposición por aprender, es menester partir de un mayor conocimiento del sujeto de la educación en el mundo actual. Existe un importante cuestionamiento respecto a quiénes son hoy en día los y las adolescentes que entran a la escuela secundaria, por lo que se requiere explorar «una cartografía mínima del mundo que viven los adolescentes» (Aguayo, 2015, p. 14), lo que implica incursionar en su identidad y vivencias cotidianas, siendo el foco de nuestro estudio lo concerniente a su experiencia en la institución escolar.

En la metasíntesis de 20 investigaciones de Acosta-Silva (2017) se cuestiona el concepto del adolescente nativo digital y la idea de Prensky (2001) que la pérdida de sentido ante la escuela se debe a que ésta no ha transformado su enseñanza acorde a la dinámica de la sociedad digital. El análisis conjunto de dichas investigaciones conduce a que no hay claridad de lo que es un comportamiento digital competente, ni se explora éste directamente, sino la percepción que los adolescentes, y que la hipótesis de la superioridad en el dominio de las TIC en los nativos digitales no se soporta empíricamente, pero esto puede deberse a que «no es que los participantes no tengan competencias digitales, sino que las que hemos medido hasta el momento no son las que ellos efectivamente tienen y valoran» (p. 485). En el estudio de Cassany y Hernández (2012), a pesar de poseer un capital letrado y plurilingüe en la red, manejar recursos retóricos variados de tipo narrativo, dominar programas informáticos de diseño y edición así como conducir proyectos propios, estos saberes no parecen apoyar a los jóvenes a aprobar las asignaturas escolares, puesto que sus docentes les demandan otro tipo de artefactos escritos. Ahora bien, el sentido del aprendizaje y la agencialidad del aprendiz resultan mayores en las prácticas vernáculas que realiza en la red, por cuenta e interés propio.

Son muchos los niños y jóvenes que, debido a sus condiciones socioeconómicas y culturales, no logran aprovechar los beneficios de la educación pública, porque no se contemplan los contenidos y estrategias pertinentes para su atención. Las comunidades con alto riesgo de vulnerabilidad social

$1 \quad$ Existe un amplio corpus de investigaciones psicológicas y sociopedagógicas enfocadas a establecer la relación entre el sentido del aprendizaje escolar y la identidad, expectativas, interacción y experiencia de vida de niños y adolescentes de distintos sectores sociales, con niveles de rendimiento escolar o de grupos culturales muy diversos. Su revisión puntual escapa a este trabajo, pero para fines de entender la importancia del sentido, en cuanto a la disposición por aprender, la motivación por el estudio, la identificación con la institución escolar o la importancia de la relación entre pares y entre docente-alumno en dicha construcción de sentido hacia la escuela y el aprendizaje, tomamos como referente a autores de la corriente sociocultural, como Marchesi (2004), Ortega, Del Rey y Córdoba (2010), mientras que en cuanto a la comprensión del desfase entre la experiencia del mundo digital de los adolescentes y las demandas de la institución escolar de nivel medio, consultamos a Cassany y Hernández (2012). 
y económica son las más propensas a presentar un alto índice de reprobación, situación que viene acompañada de falta de pertinencia de modelos educativos, carencia de autonomía de los centros educativos y ausencia de estrategias adecuadas que permitan la mejora de la calidad educativa con este tipo de poblaciones.

Como evidencia de lo antes afirmado, en uno de los reportes de la Ocde (2016), basados en los resultados de la prueba Pisa, se colocó a México entre los 20 países que tienen más porcentaje de alumnos de 15 años de edad sin el nivel mínimo en matemáticas, lectura y ciencia. Al mismo tiempo, los resultados indican que son los estudiantes de escasos recursos, los que asisten a escuelas rurales, los de origen inmigrante y las mujeres que sufren discriminación, los que tienen mayor probabilidad de dar resultados de bajo rendimiento o abandonar la escuela. Por su parte, Urrutia de la Torre y Martín del Campo (2015, p. 64), indican que en México sólo el 72\% de los jóvenes que ingresaron a la educación secundaria en la generación 2008-2011 terminaron en tiempo y forma dichos estudios, según lo programado por el sistema educativo (356 195 estudiantes se rezagaron durante el trayecto). En datos absolutos, los estudiantes de tercero de secundaria inscritos en el año escolar 2010-2011 eran cerca de dos millones (1 918 904), y al concluir el mismo, habían reprobado o desertado 86706 alumnos. Cuando se analiza el porcentaje de estudiantes que reprueban en secundaria en relación con los que abandonan los estudios, en 2015 la reprobación fue de $11.2 \%$ y el abandono de $4.7 \%$.

La representación social y las teorías que explican el fracaso escolar han cambiado sustancialmente en los últimos años. No sólo se trata de déficits cognitivos o neurológicos, de falta de esfuerzo e interés imputables al sujeto de la educación. De acuerdo con Marchesi (2004), los niños y jóvenes considerados como «malos estudiantes», representan identidades de lo más heterogéneo, sus perfiles son diferentes y necesitan respuestas educativas específicas, personalizadas. Lo que comparten es un historial de fracaso escolar y reprobación reiterada, con riesgo de abandono escolar, por lo que hay que revisar el tipo de relaciones que se establecen con el conocimiento, la manera en que aprenden, sus identidades y trayectorias personales.

Partimos de la premisa de Rudduck y Flutter (2007), quienes afirman que cuando damos voz a los estudiantes, estamos abriendo la puerta a la posibilidad de comprender y de mejorar la escuela. Hay que permitir que sean los propios actores quienes den cuenta de sus vivencias; afirman que se tiene que modificar el prejuicio que existe respecto a los niños, las niñas y el estudiantado joven, porque se les suele minimizar y ver como personas «inmaduras», con poca capacidad para opinar, tomar decisiones o emprender iniciativas para la solución de los problemas que afectan su vida y su comunidad escolar. En sus estudios con estudiantes ingleses, encuentran que aquellos que han sufrido marginación suelen ser más críticos y activistas, mientras que los que han experimentado el éxito académico, resultan menos reformistas y que tienen puntos de vista y lenguajes distintos respecto a la institución escolar (Rudduck \& Flutter, 2007, p. 144). El reto es lograr entablar el diálogo empático entre las narrativas, sin que ello cancele el disenso o el reconocimiento de conflictos, que resultan la ocasión de plantear nuevos cursos de acción.

Por su parte, en el contexto colombiano, Alvarado, Ospina-Alvarado y Sánchez-León (2016) postulan que hay que impulsar el reconocimiento y comprensión de los contenidos y formas mediante las cuales los niños y niñas que han experimentado situaciones de conflicto armado, narran sus identidades y subjetividades, puesto que de esa manera se puede comprender la construcción de sus sentidos, identidades y subjetividades particulares. Las narrativas generativas de estos niños y niñas, de sus familias y docentes, les han permitido participar como sujetos políticos, en pos de la paz y la reconciliación en sus comunidades.

Consideramos que los y las estudiantes de secundaria, tienen mucho que decir respecto a sus emociones y aprendizajes en el contexto escolar, a sus propios retos, limitaciones y capacidades, sobre el tipo de centro escolar al que les gustaría asistir, así como reflexionar sobre su identidad como estudiantes y adolescentes. Ya Lambert (2010) ha argumentado que de esta forma se hacen visibles y audibles las narrativas alternativas de los colectivos tradicionalmente silenciados, lo que permite a la par cuestionar las narrativas hegemónicas instauradas en torno a ellos. 


\section{Propósitos del estudio y pregunta de investigación}

Con esta investigación se pretendió, en primer término, contribuir al conocimiento de la identidad de los y las adolescentes considerados por la institución escolar como «malos estudiantes»o «problemáticos» debido a su bajo rendimiento escolar o a su mal comportamiento, a fin de otorgarles voz para entender, desde su propia perspectiva, cómo han vivido la experiencia escolar en la escuela secundaria a la que asisten y de qué manera esto les ha conducido a una construcción identitaria respecto a sus capacidades como estudiantes.

En segundo término, se buscó que las narrativas autobiográficas funcionaran como dispositivos pedagógicos, en cuanto «artificio complejo, pensado y/o utilizado para plantear alternativas de acción» (Sanjurjo, 2009, p. 32). Para tal finalidad, se realizó un taller de Tutoría donde en colectivo se compartieron y analizaron las narrativas y se generó una serie de relatos digitales que buscaban propiciar la reflexión sobre la identidad del estudiante y la comprensión de su situación escolar y cómo mejorarla.

De esta forma, la pregunta general de la investigación es la siguiente: ¿Es posible desvelar los factores asociados al desvanecimiento progresivo del sentido de experiencia y del aprendizaje escolar en un grupo de adolescentes de secundaria en situación de vulnerabilidad académica y social a través de sus relatos autobiográficos identitarios?

Para dar respuesta a este cuestionamiento, se procedió a la exploración de los fondos de identidad plasmados en las vivencias de adolescentes mexicanos de una secundaria pública con historia de reprobación escolar y comportamiento disruptivo, quienes plasmaron desde su propia voz y significados, mediante la construcción de relatos autobiográficos digitalizados, aquellos factores implicados en el sentido del aprendizaje y vivencia escolar. En particular, tomando en cuenta las categorías que conforman los fondos de identidad propuestos por Esteban-Guitart (2012) se indagó el tipo de ambientes educativos que les resultan estimulantes o poco motivantes, las personas o redes de apoyo con que cuentan, sus expectativas en torno a lo que esperan de su escuela, algunos de los incidentes que han resultado importantes o los han llevado a cuestionar a la institución escolar y a sus actores.

\section{Adolescentes en situación de vulnerabilidad}

En la dirección que apunta Baquero (2006), decidimos no instalar la sospecha del no aprendizaje en los estudiantes con una historia de fracaso escolar, de quienes se suele decir que no reúnen los requisitos necesarios (familiares, socioeconómicos, culturales o personales) para resultar sujetos educables que aprenden exitosamente en la escuela, de ahí que se les etiquete en las escuelas e incluso en sus familias como ineducables, desde la hora y punto que «no aprenden» los contenidos prescritos en el currículo escolar. Es así que desde la representación social de los «malos estudiantes» se les niega la educabilidad o capacidad de aprender, debido a que no son portadores de lo que se espera como condiciones supuestamente «normales» que propician el aprendizaje (motivación instrínseca, inteligencia, buena capacidad lectora, estrategias efectivas para aprender, ajuste a las normas escolares, obediencia y disciplina, etc.). De ahí que en la institución escolar se suele etiquetar a muchos estudiantes con «problemas de aprendizaje», bajo el presupuesto, por lo general no confirmado, de que adolecen de algún déficit cognitivo o neurológico que les impide apropiarse del conocimiento, sin que exista una discapacidad intelectual manifiesta, siendo el principal indicador la reprobación de materias (Marchesi, 2004).

La capacidad de aprender de los escolares, como la de los seres humanos en general, debe ser entendida como el resultado de la acción educativa en contexto y no sólo como la habilidad o déficit de un individuo particular o debido exclusivamente a su procedencia de un medio social determinado. De ahí la importancia del concepto de «educabilidad», como plasticidad o ductilidad del individuo 
para modelarse y transformarse por la influencia de múltiples estímulos, agentes y dispositivos educativos (Baquero, 2006).

Por lo anterior, en este trabajo preferimos hablar de estudiantes o adolescentes en situación de vulnerabilidad escolar o académica y en riesgo social, no de alumnos fracasados, de menores con incapacidad o problemas para aprender, ni de estudiantes conflictivos, con el fin de no etiquetarlos ni depositar únicamente en el sujeto de la educación el problema o el déficit que impide acceder al aprendizaje escolar o a la convivencia armónica con los otros. Por el contrario, postulamos que los factores asociados al desinterés por la escuela, al bajo rendimiento escolar o a un comportamiento disruptivo, resultan complejos, son fenómenos multideterminados, no unicausales, sino sistémicos. Cuando se dice «en situación de» queda abierta la posibilidad de transformar dicha situación, considerando la actuación del propio sujeto, así como la de otros actores educativos e instancias sociales.

Al hablar de la identidad del adolescente o del joven, cuando empleamos la etiqueta de «lo juvenil» estamos frente a un concepto relacional, históricamente construido, situacional y representacional, donde «se dan procesos de disputa y negociación entre las heterorrepresentaciones y las autopercepciones de los mismos jóvenes» (Esteinou, 2005, p. 31). La identidad del adolescente o del estudiante sólo puede entenderse atendiendo a su doble génesis, histórica y biográfica, ya que cobra significado gracias a la participación de los seres humanos en la cultura, en un momento histórico y territorio determinado. La construcción de la identidad implica la capacidad de autorreconocerse y atribuirse a sí mismo determinados rasgos o comportamientos, de conformar una memoria autobiográfica, sujeta a interpretación y reconstrucción, pero es una empresa eminentemente social (Bruner, 2004; Esteban-Guitart, 2012). Por otro lado, no consideramos a la identidad como una entidad esencialista o inamovible; de acuerdo con Paul Ricoeur (2006), la narrativa de vida de una persona es constitutiva de su identidad personal, en términos de la descripción del tiempo humano y de su situación, procediendo de forma dinámica y constructiva:

Hago hincapié en esta expresión de identidad narrativa porque lo que llamamos subjetividad no es ni una serie incoherente de acontecimientos ni una sustancia inmutable inaccesible al devenir. Este es, precisamente, el tipo de identidad que solamente la composición narrativa puede crear gracias a su dinamismo. (Ricoeur, 2006, p. 21)

Cuando hablamos de adolescentes, el concepto alude a agentes con identidad y agencia social, en la medida en que se apropian o rechazan conocimientos, valores, prácticas sociales, formas de ver la vida, en diversos contextos institucionales (familia, escuela, grupos religiosos, deportivos, políticos, etc.) Dichos contextos institucionales permiten que la persona se visualice a sí misma y se defina con un sentido de pertenencia, configurando de manera dinámica y relacional su propia identidad.

De acuerdo con Esteinou (2005), el individuo joven en nuestra sociedad contemporánea enfrenta, con o sin intención, una importante demanda social: busca encontrar respuestas para configurar una identidad y un proyecto de vida, tal vez imitando, confrontando o bien asimilando la cultura a su alrededor. Para comprender lo que llamamos identidad de los adolescentes (o más bien identidades), habrá que concretizar a qué adolescentes nos referimos y ahondar tanto en sus mentalidades y prácticas como en el contexto situado en que se desarrollan:

De hecho, no hay «juventud» sino juventudes. Se trata de una condición históricamente construida y determinada, depende de diferentes variables, siendo las más notorias la diferenciación social, cultural, el género y la generación. (Margulis, 2001, p. 42)

\section{Identidad narrativa y fondos de identidad}

Desde una perspectiva sociocultural, la identidad implica un proceso dinámico de construcción de narrativas personales, autobiográficas, donde se habla de la definición que uno o una hace sobre sí mismo o sí misma (la vivencia, reconocimiento e interpretación que se tiene sobre sí) a través de una historia de vida o narración que provee sentido y unidad a los sucesos vitales (Bruner, 2004). Al 
elaborar un relato sobre su propia historia, el o la estudiante piensa sobre sí mismo y sus experiencias de vida; puede reflexionar en torno a que los acontecimientos personales representan una unidad biográfica, pero no estática, porque no se trata sólo del pasado o de lo que le ha sucedido, sino de lo que se puede o quiere llegar a ser. Además, si se comparten y analizan los relatos con los demás miembros de la clase, puede ocurrir un proceso de proyección (interacción e integración con otros agentes sociales) para asumirnos como parte de un colectivo humano (Herreros, 2012).

La identidad tiene un componente fenomenológico, dado por la expresión personal de los propios estados introspectivos (vivencias y emociones del sujeto), pero en coexistencia con mecanismos de intersubjetividad, dada la importancia de las interacciones que ocurren en contextos y situaciones determinadas. Por ende, la identidad no sólo es subjetiva, sino también es cognición situada, intersubjetividad, producto de la interacción social con distintos agentes. Puede afirmarse que la identidad se construye a través del lenguaje y la práctica social en distintas comunidades, como reflejo de la cultura.

Desde una mirada analítica y ecológico-sistémica², Esteban-Guitart (2012) postula un concepto central para este trabajo: los fondos de identidad, que son los recursos históricamente acumulados, culturalmente desarrollados y socialmente distribuidos y transmitidos, esenciales para la autodefinición, autoexpresión y autocomprensión de las personas. Cuando se exploran los fondos de identidad de un sujeto, suelen aflorar los fondos sociales, es decir, aquellas personas que considera más significativas y que se convierten en recursos para su desarrollo, así como las prácticas de identidad o actividades que uno realiza y que suelen ser las más recurrentes e importantes (como asistir a clases, practicar un deporte, ejecutar cierto instrumento musical o navegar en internet). La utilización de artefactos culturales es nodal para autoexpresarse o autodefinirse, pues se relaciona con los instrumentos (físicos o semióticos) que empleamos en el día a día en la mediación del pensamiento y la actividad. Los fondos de identidad geográficos se relacionan con aquellos espacios físicos o territorios (por ejemplo, nuestro barrio, la escuela a la que asistimos) donde nos autodefinimos como parte de un colectivo, mientras que los fondos institucionales de identidad desvelan la influencia de determinada ideología o normas de conducta (por ejemplo, la forma en que concebimos y vivimos el trabajo escolar).

Dado que existen tanto desajustes como continuidades-discontinuidades en los procesos de construcción de la identidad de una persona, los fondos de conocimiento de las instituciones en que participa un adolescente (habitualmente la escuela y la familia) pueden ser o no coincidentes en comparación a lo que él o ella internalizan. La identidad está en constante revisión, «fluye» en cada nuevo contexto, por lo que cada uno puede expresar distintas identidades, no siempre conectadas ni necesariamente coincidentes. Por ello, existe la polémica de si sería más apropiado hablar en plural de las distintas identidades que re-construye una misma persona, dados los contextos y experiencias en que participa a lo largo y ancho de la vida. Por ende, es difícil, si no imposible, hablar de identidad como constructo homogéneo o libre de tensiones y contradicciones.

\section{Metodología}

\section{Tipo de estudio}

Se condujo una investigación cualitativa, biográfico-narrativa, que intenta aportar a la investigación didáctica a través de desvelar los significados de las historias de vida de los actores educativos (Bolívar, Domingo, \& Fernández, 2001). Este enfoque destaca la importancia de la subjetividad, las emociones y la experiencia fenomenológica; afirma que el significado viene dado por la autointerpretación que los sujetos relatan en primera persona, pero siempre en un contexto relacional.

2 La perspectiva ecológico-sistémica del psicólogo ruso Urie Brofenbrenner (1987) postula que el desarrollo humano y la sociedad se desenvuelven en los planos cognitivo, moral y relacional en función de los ambientes o contextos en que viven. Propone una visión sistémica, naturalística y analítica de los macrosistemas, mesosistemas, exosistemas y microsistemas donde se desenvuelven los colectivos humanos, destacando la escuela, el hogar, el barrio, entre otros, como ambientes de clara influencia en la constitución de la conducta e identidad humana. 
Con base en Amar-Rodríguez (2016), en una investigación narrativa se parte del principio freiriano de «leer el mundo»: comprender una realidad en la que estamos inmiscuidos, mostrando una visión personal ante una problemática que atañe a un informante, en este caso, a un grupo de adolescentes de secundaria. La investigación narrativa, dada su cualidad interpretativa, busca descubrir lo que dice y siente nuestro informante; no es un estudio del sujeto, sino la posibilidad de desvelar su subjetividad y darle voz. Implica una mirada poliédrica, de narración de lo vivido, de conocimiento situado, donde «no buscamos la veracidad de los hechos, sino la coherencia interna de lo narrado dentro de su cotidianeidad» (Amar-Rodríguez, 2016, p. 980).

\section{Contexto y participantes}

El estudio se realizó en el ciclo escolar 2016-2017 en la asignatura Tutoría (Secretaría de Educación Pública, 2011). Se trabajó en una dinámica de taller y asesorías, durante 50 minutos a la semana, en la Escuela Secundaria Técnica 101, Delegación Iztapalapa, en una de las zonas más marginadas y con mayor incidencia delictiva en Ciudad de México. ${ }^{3}$

El nivel socioeconómico de los estudiantes oscila entre medio-bajo y bajo en relación con ingresos y escolaridad de los padres. La zona que rodea al plantel presenta problemas de narcomenudeo y violencia, mientras que al interior de la escuela se reporta el incremento de embarazos adolescentes y de acoso escolar. El subdirector ha emprendido campañas para que las instaciones se encuentren limpias y libres de graffiti, para exigir la portación del uniforme escolar y el cumplimiento del reglamento escolar. En la misma dirección, y ante los pobres resultados académicos de la población, propuso la conformación de un grupo especial de tutoría académica dirigido a los alumnos con mayor riesgo académico, quienes estaban propensos a abandonar la escuela y a no concluir la educación secundaria, siendo ésta la población-meta del estudio. ${ }^{4}$

Participaron 33 estudiantes de tercer grado de secundaria, ocho mujeres y 25 hombres, con una edad promedio de 15 años. Para autoridades y docentes, eran adolescentes rebeldes, poco interesados en estudiar, que causaban problemas en el aula, no entregaban tareas y reprobaban los exámenes. Algunos ya habían sido expulsados o suspendidos, tenían varias materias reprobadas y reportes de mala conducta. En la mayoría de los casos, se había solicitado la intervención de los padres, con poco éxito.

Para nosotros, se trataba de adolescentes en situación de vulnerabilidad académica y social, debido a factores personales, familiares, socioeconómicos y escolares muy diversos, que fueron explorados a través de entrevistas personales y mediante la revisión de sus expedientes académicos. La problemática venía presentándose de tiempo atrás y a las autoridades les preocupaba que no lograran

$3 \quad$ Iztapalapa es la demarcación de la Ciudad de México donde más delitos de alto impacto se cometen. Según cifras del Secretariado Ejecutivo del Sistema Nacional de Seguridad Pública, de enero a noviembre de 2017 ocurrieron 212 asesinatos en la demarcación, que ha tenido el primer lugar en número de homicidios en los últimos tres años. Es la delegación donde se denuncian más delitos como homicidio doloso, lesiones dolosas por disparo de arma de fuego, secuestro, violación, robo a casa-habitación con violencia, robo de auto, robo a transportista, robo a transeúnte con violencia, robo a pasajero en transporte público, robo a negocio, robo a cuentahabiente. Del total de delitos que ocurren en Iztapalapa diariamente $19.9 \%$ son con violencia, es decir, dos de cada 10 son de alto impacto (Cruz, 2018).

De los cuatro mil puntos móviles y fijos de venta de droga, según el jefe de la Policía Judicial del Distrito Federal, la mayoría se encuentra en Iztapalapa y Venustiano Carranza. El avance del narcomenudeo en Iztapalapa es alarmante: de los 100 puntos de ventas que existían en el año 2000, pasó a cerca de 800 en 2007 y ha continuado en incremento. El narcomenudeo invade las secundarias y es un gran atractivo de trabajo para los jóvenes de familias pobres (Balboa, 2007).

De acuerdo con la Encuesta de Consumo de Drogas en Estudiantes 2012 en la ciudad de México, elaborado por el Instituto Nacional de Psiquiatría (INP) y el Instituto para la Atención y Prevención de las Adicciones (Iapa), se establece que en Iztapalapa predomina el consumo de drogas tanto lícitas como ilícitas. Entre estudiantes de secundaria el 29.8\% de los encuestados consumió alcohol el último año; tabaco 9.4\%; marihuana 5.9\%; e inhalables 5.3\%. Se encontró que a la temprana edad de 9 años hay niños y niñas consumiendo solventes (Alarcón, 2014).

4 La información fue proporcionada por el Director y el Subdirector del plantel, que formaron el grupo de estudiantes. La responsable de la Tutoría, segunda autora de este artículo, corroboró la información en los expedientes de los y las adolescentes, y mediante una entrevista semiestructura que realizó con cada participante al inicio del estudio. En algunos casos se tuvo acceso a información adicional a través de padres, tutores o docentes. Cabe mencionar que el grupo era etiquetado en el plantel como «los menores infractores», calificativo mal empleado que sin embargo da cuenta de la percepción social generada.En las entrevistas se reportaron situaciones de autolesiones, consumo de sustancias ilícitas, acoso escolar, violencia familiar, abuso sexual, entre otras. En todos los casos tenían al menos tres asignaturas reprobadas y algunos jóvenes habían sido expulsados temporalmente por problemas de comportamiento en función de las sanciones que impone el reglamento escolar. 
concluir el ciclo escolar con calificaciones aprobatorias para obtener el certificado de secundaria. No estaban recibiendo tutoría ni asesoría pedagógica, sólo una estudiante acudía al psicólogo y otro más recibía medicación psiquiátrica.

La realización de las narrativas autobiográficas fue el primer paso de una intervención más amplia, que se diseñó bajo los principios de la personalización del aprendizaje (Coll, 2016), con la intención de explorar la identidad de los adolescentes, para fortalecer su agencia personal en función de sus necesidades y características, dotarlos de estrategias de aprendizaje independiente con el apoyo de tecnologías digitales y mejorar sus entornos personales para aprender, vinculando aprendizajes formales e informales. La intención era instaurar una mirada más sistémica y no peyorativa de sí mismos, promover la identificación y comprensión de una diversidad de factores y situaciones que operan en la construcción de su identidad y en su vivencia educativa.

En todos los casos, se trabajó en una dinámica de taller colectivo, para delimitar un trayecto personal de aprendizaje, capacitar a los y las participantes en estrategias de aprendizaje y estudio independiente relacionadas con los contenidos curriculares, en la promoción de una mayor motivación instrínseca y en el esclarecimiento de un proyecto de vida. De particular interés resultó el apoyo socioemocional a los adolescentes. En este artículo se reporta lo relacionado con el primer ciclo de trabajo: la elaboración y digitalización de sus relatos personales donde expresan sus fondos de identidad en función de cómo viven la escuela secundaria.

\section{Procedimiento}

La etapa de conocimiento de la identidad del estudiante de secundaria, consistió en generar un relato autobiográfico en forma de narrativa testimonial corta, que diera cuenta de algún suceso relevante para los estudiantes en su trayecto en la escuela secundaria y donde además de contar la anécdota, hubiera una reflexión o sentido profundo sobre el mismo. La metodología empleada se basó en Lambert (2010) respecto a la elaboración de relatos digitales personales (digital storytelling en inglés), y se siguió la ruta de diseño tecnopedagógico propuesta en Contar historias. Relatos digitales personales (en http://grupogiddet.wix.com/rdpgiddet).

Los participantes eligieron libremente el tema y elaboraron narrativas personales, donde compartieron alguna experiencia relevante sobre sus vivencias en la escuela; éstas fueron grabadas en audio en sus teléfonos celulares. Posteriormente, en una dinámica de taller de trabajo, los estudiantes se agruparon en equipos para compartir sus narrativas y se realizaron plenarias para discutir el sentido de sus experiencias y lo que ello aportaba en términos de sus fondos de identidad. Se les explicó en qué consiste la estructura de una narrativa y se les asesoró en la mejora de los relatos. Tomaron fotografías en distintos espacios del plantel educativo, grabaron clips y bajaron audio e imágenes de la red, alusivos al contenido de la narrativa para su ensamble como producción multimedia. Aprendieron el manejo de la herramienta digital Stupeflix (https://studio.stupeflix.com) para crear videos personales.

Aunque todos los estudiantes aprendieron con facilidad y buena disposición la mecánica del uso del software así como el proceso de digitalización y musicalización, el equipo de cómputo de la escuela resultó poco funcional, lento y con fallas en internet para la edición multimedia requerida. Debido a esto, cada equipo seleccionó sólo una de las narrativas de sus integrantes para convertirla en video, tomando en cuenta aquellas situaciones con las que más se identificaron y consideraron comunes. A partir de este momento, los videos se trabajaron colaborativamente.

\section{Resultados}

La mayor parte de las vivencias relatadas por los adolescentes se refieren a incidentes críticos vividos en el plantel, es decir, relatan situaciones problemáticas que les han causado dudas, perplejidad, malestar, sorpresa, y que se traducen en una valoración emocional negativa del suceso. Lo anterior no es de extrañar, en cuanto Bruner (2004) plantea que la centralidad del conflicto es la esencia de la narrativa y que una historia digna de ser contada desvela experiencias vitales inesperadas, que 
rompen los cánones establecidos. En esto reside la posibilidad de convertir los relatos en instrumentos de cuestionamiento y reflexión. Por otro lado, era esperable que afloraran aquellas situaciones problemáticas en que se ven inmersos estos adolescentes, dado que su experiencia escolar no ha redundado en éxito escolar ni en aceptación en la comunidad, lo cual coincide con lo encontrado por Rudduck y Flutter (2007).

En la Tabla 1 se indican las principales dimensiones de análisis del contenido de las narrativas en función de su interés para los propios participantes y de los temas centrales que ellos resaltan en la trama. Son dimensiones que atraviesan varias narrativas, aunque en algunas son el foco principal. En todos los casos su relación es directa o por lo menos influye con la situación de fracaso escolar y los problemas de comportamiento de los estudiantes, tomando en cuenta la percepción de los participantes en las discusiones realizadas en el taller. Al mismo tiempo, puede decirse que las categorías que propone Esteban-Guitart (2012) para explicar los fondos de identidad (espacios físicos, redes sociales de apoyo, prácticas socioculturales, valores institucionales) aparecen en mayor o menor medida en todas las narrativas (Los videos se encuentran alojados en http:/grupogiddet.wixsite.com/rdpgiddet/ vivir-mi-secundaria y también pueden recuperarse en YouTube).

Tabla 1. Ejemplo de relatos digitales del proyecto «Vivir la secundaria»

\begin{tabular}{|l|l|c|}
\hline \multicolumn{1}{|c|}{ Nombre del relato } & \multicolumn{1}{c|}{$\begin{array}{c}\text { Dimensiones de identidad y sentido de la experiencia escolar } \\
\text { que explora la narrativa. }\end{array}$} & $\begin{array}{c}\text { Dirección electrónica } \\
\text { de acceso libre }\end{array}$ \\
\hline $\begin{array}{l}\text { «Se puede ser feliz en } \\
\text { la secundaria? » por } \\
\text { aka Mei Tsumabuki }\end{array}$ & $\begin{array}{l}\text { Aspectos socioemocionales de la vida de una adolescente. } \\
\text { Problemática de lesiones autoinflingidas («cutting») como resultado } \\
\text { de sentir rechazo y ser objeto de acoso escolar. Importancia de la } \\
\text { autoestima y la resiliencia. }\end{array}$ & $\begin{array}{c}\text { https://youtu.be/ } \\
\text { vVF2-7Z2sc }\end{array}$ \\
\hline $\begin{array}{l}\text { «Buenas amistades», } \\
\text { por Las mejores } \\
\text { amigas. }\end{array}$ & $\begin{array}{l}\text { El valor de la amistad y la aceptación de los pares. La regulación } \\
\text { identidad en la interacción con los otros (la intersubjetividad). }\end{array}$ & $\begin{array}{c}\text { https://youtu.be/ } \\
\text { y2hEGFh5AxY }\end{array}$ \\
\hline $\begin{array}{l}\text { «Jarabullying», por } \\
\text { Bocanegra y Anyelo }\end{array}$ & $\begin{array}{l}\text { La interpretación que dan los jóvenes al acoso escolar. La relevancia } \\
\text { de la empatía y la emoción moral. La normalización de la violencia. } \\
\text { La importancia de repensar la educación para la convivencia. }\end{array}$ & $\begin{array}{c}\text { https://youtu.be/ } \\
\text { hZ9BEf90lbs }\end{array}$ \\
\hline $\begin{array}{l}\text { «Lo intrascendente» } \\
\text { por Bistek.2 y Chulo } \\
\text { Bistek }\end{array}$ & $\begin{array}{l}\text { Lo que molesta a los adolescentes en su escuela: la forma en que } \\
\text { se les avergüenza públicamente, el manejo poco transparente de las } \\
\text { cuotas que les exigen, la forma en que se pretende educarlos. La } \\
\text { relación con los agentes educativos. }\end{array}$ & https://youtu.be/ \\
\hline $\begin{array}{l}\text { TGLv701S3y4 } \\
\text { «U.N.I.F.O.R.M.E.S. }\end{array}$ & $\begin{array}{l}\text { La existencia de prácticas y normativas en las que no son tomados } \\
\text { en cuenta. Las inconsistencias e inequidad en el reglamento del } \\
\text { uniforme escolar y la negativa a permitir la expresión personal en la } \\
\text { propia vestimenta. }\end{array}$ & $\begin{array}{l}\text { https://youtu.be/ } \\
\text { un54OyI-MDg }\end{array}$ \\
\hline
\end{tabular}

\section{Aspectos socioemocionales}

Los aspectos socioemocionales ${ }^{5}$ de la vida de los adolescentes fueron los que recibieron más menciones en las narrativas y resultaron los más significativos en torno a la conformación de la identidad y la autovaloración. Prácticamente todos los estudiantes narraron incidentes donde hablan con sus propias palabras de cómo perciben e interpretan la expresión de sus emociones en el entorno

$5 \quad$ Para efectos de este trabajo consideramos que «Las emociones son el conjunto de percepciones, interpretaciones y respuestas fisiológicas a una situación dada (sea esta real o imaginada). Es el lugar donde lo biológico y lo cultural entran en contacto, donde una alteración físico-psicológica adquiere un nombre y se convierte en amor, deseo, miedo o enfermedad según los códigos culturales en los que la persona que la sienta esté inscrita. Después, el conjunto nombrado se valora, se pule y se comunica a los otros también según códigos culturales» (Manrique, 2015, p. 802). Evitamos una concepción narcisista, universalista, etiquetante o patologizante del tema, permitiendo que los y las adolescentes expresaran con su propia voz dichas percepciones e interpretaciones, y fueran ellos quienes asignaran su valor positivo o negativo. Por supuesto que su propia valoración es resultado de la educación emocional que han recibido y tiene un fuerte correlato cultural. 
escolar, siendo la aceptación o rechazo de los pares lo que más impacto les genera. Aparece asimismo el correlato emocional de la relación pedagógica e interpersonal con docentes y autoridades, así como su inconformidad con la normativa escolar y a las formas de enseñanza.

Los adolescentes narraron la reacción y manejo de sus emociones ante situaciones de maltrato y acoso, los problemas de valoración personal que han experimentado, los comportamientos de otras personas que los han lastimado, las «etiquetas» que les han impuesto, su deseo de ser aceptados entre sus pares. Las emociones que consideran «negativas» (miedo, enojo, frustración, etc.) se encuentran ligadas a un bajo aprovechamiento escolar, al desinterés por los estudios, y ellos mismos discuten que no necesariamente existe una falta de capacidad para aprender. Dependiendo de su estado emocional y de la capacidad de regulación del mismo, de autocontrol e interacción con los otros, es que se despierta el interés por aprender o la disposición por estudiar. En algunos jóvenes el argumento se encuentra en torno a la resistencia a lo que perciben como imposición o autoritarismo de parte de los agentes educativos.

Asimismo, narran las emociones «positivas» (felicidad, alegría, satisfacción, agrado, etc.), con relación a estrategias y personas que les han ayudado a superarse como estudiantes y como seres humanos; entre ellos, sus mejores amigos o amigas, algunos docentes o tutores, y a veces, sus padres. En particular, el tema de la aceptación de los pares y su influencia en su propio comportamiento, desempeño y autovaloración, es una constante en los relatos. Dos relatos digitales que enfatizan los aspectos socioemocionales y la importancia de los pares en la vida de estos los adolescentes son los siguientes:

- «¿Se puede ser feliz en la secundaria?» por aka Mei Tsumabuki (seudónimo de la autora del relato), donde la estudiante narra que el rechazo de sus compañeros de «fue un golpe muy bajo para mí y, como cualquier adolescente haría, me autolesionaba, es decir que me lastimé... ya no sentía el interés, incluso llegué a asistir con un psicólogo para tratar los temores de un adolescente que es aislado y piensa que estará solo, que es un inútil, que no vale nada». La estudiante es una adolescente que destaca por su capacidad para la escritura reflexiva y creatividad artística como dibujante, como lo demuestra en su narrativa, que quiso elaborar individualmente. No obstante, narra que desde niña no se siente aceptada por sus compañeros de escuela, ha sido objeto de rechazo y burlas, por lo que al arribar a la adolescencia bajó su rendimiento escolar y comenzó a practicarse «cutting» (autolesiones con navajas en piernas y brazos). En la narrativa, esta adolescente habla explícitamente de su baja autoestima y de la importancia y esfuerzo por superar esta problemática, que asocia con su bajo desempeño y desinterés escolar.

- «Buenas amistades», por Las mejores amigas, refleja la intensidad y labilidad con que se viven las relaciones afectivas y la gran influencia de los pares en esta etapa, en el plano personal y el académico. Refleja que la identidad se construye en la interacción con los otros significativos, en cuanto destaca la importancia de las redes sociales y la aceptación del grupo de pares. También plantea la importancia de la educación socioemocional de los adolescentes, dada la necesidad de estrategias de regulación crítica de las emociones y de interacción dialógica para resolver conflictos.

\section{Acoso escolar y violencia}

- «Jarabullying», por Bocanegra y Anyelo. Los adolescentes tienen una representación distinta a la de los adultos respecto al bullying, sobre todo si son parte del grupo de agresores. Para muchos de ellos, no implica un daño «serio» para aquellos que reciben las agresiones físicas o verbales, éstas son parte de rituales y formas de relación («así nos llevamos aquí», «al nuevo se le da su bienvenida»). El discurso adulto no parece disuadirlos de estas prácticas y la respuesta de la autoridad escolar se focaliza en el castigo, no en la reparación del daño. 
La manifestación de alguna emoción o reflexión moral ocurre sólo cuando experimentan empatía, debido a que alguna persona cercana y significativa ha recibido acoso. En este relato un grupo de varones acosa a Jaramillo y a Iñaki, uno de ellos es el alumno «nuevo», el otro es «gordo», lo que justifica las agresiones. No obstante, en el desenlance, aflora la emoción moral y la empatía, cuando se reflexiona cómo es que el acoso escolar afecta a la hermana de uno de los agresores. En esta escuela secundaria se encontró que el bullying es una práctica sociocultural naturalizada por los adolescentes e invisibilizada o minimizada por los adultos («no hay agresiones graves», «siempre ha sido así») aunque muchos estudiantes tienen reportes reiterados de mal comportamiento y se les castiga suspendiéndolos de la escuela por días o semanas. Se encontró que los adolescentes llegan a grabar con sus teléfonos celulares las prácticas de acoso o las riñas entre sus compañeros o compañeras para subirlos a la red, a fin de «divertirse» viéndolos y burlarse de los protagonistas. En otras narrativas los adolescentes hablan de la violencia, las agresiones y la inseguridad de su zona escolar y en el barrio donde viven, dicen sentirse inseguros, pero no parecen reconocer que ellos mismos han socializado la violencia y la practican al interior de la escuela.

\section{Autoridad de los adultos y normativa escolar}

Los estudiantes se mostraron críticos ante la forma en que se ejerce la autoridad en su escuela, ya sea por parte de directivos, docentes o personal de servicio. Hablaron de situaciones que consideran injustas, rígidas o poco trasparentes, y que los afectan directamente. También mencionaron que en muchos casos quien ejerce la autoridad y sus normas no tiene la razón, no plantea ninguna justificación o se contradice en su proceder, pero en la escuela tienen que obedecer sin posibilidad de tomar alguna decisión y sin opción a réplica. Al respecto, dos de los relatos digitales plantean dichas situaciones:

- En «Intranscendente» dos adolescentes, con el seudónimo de Bistek.2 y Chulo Bistek, dialogan respecto a lo que les molesta de su escuela. Cuestionan la manera en que se expone y avergüenza a los estudiantes, debido a sus bajas calificaciones o porque no pagan las cuotas, así como la manera en que los pretenden educar para la convivencia. Plantean que en algunos programas sociales los obligan a dar «donativos» que a fin de cuentas no son voluntarios, o les piden «cuotas» de las que no se sabe el destino de los recursos económicos. Cuestionan el ejercicio de la autoridad en el plantel, así como el que no son tomados en cuenta en muchas decisiones.

- En el relato colaborativo «U.N.I.F.O.R.M.E.S.» destaca el cuestionamiento al reglamento escolar, respecto al cual los estudiantes no están de acuerdo ni pueden opinar; por ejemplo, la exigencia de llevar tenis blancos o el que no puedan portar otras prendas de ropa que no sean del uniforme escolar. En la discusión en plenaria, dijeron que «los quieren ver a todos iguales», que no los dejan expresarse ni mostrar sus gustos o estilos, y que portar un uniforme no evita que se manifiesten las diferencias económicas entre ellos. La lógica de los reglamentos consiste en prohibir ciertas formas de ser, de arreglo personal o comportamientos que molestan a los docentes, pero los alumnos dicen que esto no les permite sentirse mejor o aprender.

\section{Condiciones de la institución escolar y formas de enseñanza}

Los alumnos reiteran en sus narrativas que las condiciones y servicios que se ofrecen en la escuela dejan mucho que desear. En el relato «Las instalaciones» una estudiante que habla con sus compañeros, propone «que sean mejoradas las instalaciones de los salones, del salón de informática como las computadoras, de los talleres, los baños para que puedan servir». En el relato «La cooperativa escolar», dos estudiantes platican que la calidad de los alimentos que se venden en la escuela es mala, 
que son costosos para ellos y poco saludables: «Siguen vendiendo comida «chatarra〉 [...] Yo creo que deberían de vender cosas más sanas y en mejor estado, hace daño a nuestra salud y si venden puras cosas chatarra, pues habrá bastante obesidad».

Asociado a la falta de buenas instalaciones, los alumnos narran la forma en que se les enseña. No hay acceso a la red en las aulas y las actividades deben realizarse de manera tradicional, con toma de apuntes, dictado y copia en cuadernos, reproduciendo lo que se escribe en el pizarrón o lo que dice el docente. Se prohíben los teléfonos celulares, aunque la mayoría los porta y usa «a escondidas». Muchos docentes no pueden controlar el comportamiento de los estudiantes en la clase, hay «muchas faltas de respeto» y la respuesta es el castigo o la reprobación. Lo que se enseña y lo que se pide como tareas no suele despertar el interés de los estudiantes, a ello atribuyen su distracción e incumplimiento.

La redacción y escritura de los relatos requirió de reiteradas revisiones y apoyos para la mejora de su sintaxis, coherencia y ortografía. Los adolescentes reportaron que casi nunca escriben sobre sí mismos y su escritura escolar es de reproducción de textos o respuesta a preguntas en cuestionarios. No obstante, gracias a la mediación en las sesiones del taller de tutoría, los estudiantes comprendieron la estructura narrativa y pudieron lograr una reflexión respecto a la problemática que relatan, así como algunas soluciones. El uso de las tecnologías digitales despertó en ellos mucho interés y disposición, sobre todo por aprender programas multimedia. Esto coadyuvó a su compromiso en el proyecto de elaboración del video.

Consideran que es a nivel de las autoridades escolares y docentes que se tienen que atender los problemas que reportan; uno de los relatos concluye así: «Y tenemos la esperanza de que este mensaje llegue a las autoridades para que puedan hacer algo».

\section{Discusión y conclusiones}

La categoría de fondos de identidad (Esteban-Guitart, 2012), permitió un acercamiento pertinente al proceso de construcción identitaria en los y las adolescentes, siendo los más relevantes en este estudio los fondos de tipo social (interacción favorable o no con personas significativas: pares, docentes y autoridades); las prácticas socioculturales (rutinas y actividades que no generan sentido ni en el aula ni en las tareas escolares); espacios físicos y artefactos culturales (instalaciones escolares y sanitarias deficientes, equipamiento ajeno a la expectativa de los estudiantes con relación al mundo digital o al tipo de actividades que les gustaría realizar) y simbólico (la expresión emocional sobre la relación interpersonal, la convivencia, el aprendizaje escolar, la normativa que define la participación y el lugar de poder en la escuela).

Al mismo tiempo, la narrativa de los adolescentes recoge el sentir colectivo respecto al ethos o forma común de vida y comportamiento de la institución escolar, al menos como lo perciben los adolescentes que no han experimentado ni el éxito académico ni la aceptación social, y que se caracteriza por un ambiente jerárquico, poco democrático y excluyente. Los resultados de este estudio, son coincidentes con otros estudios realizados con poblaciones afines (Arreola, 2017; Reyes, 2016). Sin afán de generalizar la situación, encontramos que la cultura escolar de la escuela secundaria está subordinada a prácticas educativas rutinarias y reproductivas, poco motivantes y monótonas desde la perspectiva del estudiante, centradas en ordenamientos de disciplina institucional de tipo heterónomo. Las situaciones que se valoran favorablemente y que generan disposición tienen que ver con actividades libres, elegidas por ellos, relacionadas con sus intereses y realizadas con los pares con los que tienen una relación de amistad.

La escuela opera imponiendo castigos, malas notas y reportes de mal comportamiento, pero no se atienden a fondo cuestiones como las encontradas: chicas que se autolesionan ante la imposibilidad de manejar su ira y frustración (Benassini, 2017) o chicos que acosan a sus compañeros y en quienes las lecciones curriculares de convivencia o la normatividad que sanciona el «bullying» tienen poco eco en su comportamiento, dado que no se logra incidir en sus representaciones, en sus valores ni en su emoción moral. Por el contrario, desde la narrativa de los adolescentes, la interacción positiva y la aceptación de los pares permite fortalecer a la comunidad educativa si se logran establecer vínculos 
de apoyo y protección mutua y éste puede ser un componente de resiliencia social en chicos que han experiementado vulnerabilidad y violencia (Reséndiz, 2016).

Corroboramos en el estudio la importancia de dar voz a los actores a través de relatos digitales personales, como una forma de «leer la realidad» (Amar-Rodríguez, 2016) que implica al estudiantado. En este caso ha permitido que una población poco escuchada y a la cual se le resta educabilidad y agencia (los «ineducables» según Baquero, 2006; «los malos alumnos» según Marchesi, 2004) expresen desde su perspectiva el por qué de su malestar en la escuela, lo que los ha llevado a la pérdida de sentido del aprendizaje escolar (Coll, 2016) o por el contrario, lo que puede apoyar a superar la situación de vulnerabilidad en que se encuentran y constituir factores resilientes o de protección (Feito, 2007; Reséndiz, 2016). El que se trabajara con los estudiantes desde una mirada de identidad alternativa (no como estudiantes reprobados o malos alumnos, sino como personas en situación de vulnerabilidad con posibilidad de cambio y mejora), contribuyó tanto a una buena relación pedagógica entre el grupo y los investigadores como a generar un ambiente propicio para las actividades propuestas en el taller de trabajo. Esto permite re-pensar con el colectivo de estudiantes en identidades alternativas y prospectivas, orientadas a un proyecto propio, que no tiene por qué caer en discursos simplistas, estereotipados ni triunfalistas (Manrique, 2015)

Proponemos que para apoyar a estudiantes que han tenido malas experiencias en los entornos escolares, hay que trabajar con ellos la toma de conciencia de dichas situaciones, la comprensión crítica de los factores presentes, la adquisición de habilidades para una mayor autodirección en el aprendizaje, para el manejo de las emociones y la colaboración. La intervención centrada exclusivamente en el aprendiz y su conducta de estudio es insuficiente. El fracaso escolar debe y puede atenderse (idealmente prevenirse) mediante acciones que incidan en el contexto socioeducativo, entendiendo que el aprendizaje implica acciones de índole social, cognitiva y socioemocional, que conducen a repensar la interacción con agentes educativos y pares.

Entre las limitaciones del estudio, resalta que la intervención realizada se restringió al espacio de tutoría con los participantes a lo largo de un ciclo escolar, mientras que para lograr un cambio sistémico se requiere idealmente de la participación de docentes, directivos y padres de familia, así como de la posibilidad de conformar un equipo de asesoramiento psicopedagógico continuo. No obstante, aunque esto no fue posible, los estudiantes lograron avanzar en la comprensión de su identidad, en el desarrollo de estrategias de trabajo y regulación del aprendizaje con la mediación de las tecnologías digitales, y sobre todo, en experimentar un ambiente donde sus voces fueron escuchadas.

Desde nuestra perspectiva, la narrativa autobiográfica puede fungir como un dispositivo pedagógicio para tematizar y reflexionar sobre los aspectos que suelen desestabilizar o vulnerar a los estudiantes y para planear algunas alternativas a sus trayectorias personales y proyectos de vida.

\section{Agradecimientos:}

- A la DGAPA-Unam a través del proyecto Papime PE300217.

- A la Fundación SM de México y en particular a su directora, Cecilia Espinosa.

\section{Lista de referencias}

Acosta-Silva, D. A. (2017).Tras las competencias de los nativos digitales: avances de una metasíntesis. Revista Latinoamericana de Ciencias Sociales, Niñez y Juventud, 15(1), 471-489. doi:10.11600 /1692715x.1513014062016

Aguayo, J. M. (2015). El despliegue simbólico digital de adolescentes de secundaria en Facebooky sus implicaciones en la construcción de su identidad personal. Guadalajara: UPN Jalisco.

Alvarado, S. A., Ospina-Alvarado, M. C., \& Sánchez-León, M. C. (2016). Hermenéutica einvestigación social: narrativas generativas de paz, democracia y reconciliación. Revista Latinoamericana de Ciencias Sociales, Niñez y Juventud, 14(2), 987-999. doi:10.11600/1692715x.14207170215 
SENTIDO DE LA EXPERIENCIA ESCOLAR EN ESTUDIANTES DE SECUNDARIA EN SITUACIÓN DE VULNERABILIDAD

Alarcón, J. C. (28 de mayo de 2014). No cesan los 'focos rojos' en Iztapalapa por consumo y venta de drogas. MVS Noticias. Recuperado de:

http://www.mvsnoticias.com/\#!/noticias/no-cesan-los-focos-rojos-en-iztapalapa-por-consumo-yventa-de-drogas-667

Amar-Rodríguez, V. (2016). Leer la vida. Una investigación desde la perspectiva narrativa. Revista Latinoamericana de Ciencias Sociales, Niñez y Juventud, 14(2), 975-986. doi:10.11600/169271 5x.14206261015

Arreola, L. (2017). Relación pedagógica. México, D. F.: Universidad Pedagógica Nacional.

Balboa, J. (8 de julio de 2007). Narcomenudeo, actividad comercial muy redituable para los jóvenes de El Hoyo. La Jornada. Recuperado de: http://www.jornada.com.mx/2007/07/08/index.php?se ction $=$ capital\&article $=035 \mathrm{n} 1$ cap

Baquero, R. (2006). Sujetos de Aprendizaje. Buenos Aires: Ministerio de Educación, Ciencia y Tecnología de la Nación. Recuperado de: http:/www.porlainclusion.educ.ar/documentos/ modulo2mail.pdf

Benassini, O. (2017). Niñas border. México, D. F.: Trillas.

Bolívar, A., Domigo, J., \& Fernández, M. (2001). La investigación biográfico-narrativa. Madrid: La Muralla.

Brofenbrenner, U. (1987). La ecología del desarrollo humano. Barcelona: Paidós.

Bruner, J. (2004). Life as narrative. Social Research, 71(3), 691-710.

Cassany, D., \& Hernández, D. (2012). ¿Internet: 1; Escuela: 0? CPU-e, Revista de Investigación Educativa, 14, 126-141. Recuperado de:

http://www.uv.mx/cpue/num14/opinion/cassany hernandez internet_1_escuela 0.html

Coll, C. (2009). Enseñar y aprender en el siglo XXI: el sentido de los aprendizajes escolares. En A. Marchesi, J. C. Tedesco, \& C. Coll (Coords.), Reformas educativas y calidad de la educación (pp. 101-112). Madrid: OEI Santillana. Recuperado de:

http://congreso.dgire.unam.mx/5tocongreso/Ensenar-y-aprender-en-el-siglo-XXI-Los-nuevossentidos.pdf

Coll, C. (2016). Personalización del aprendizaje escolar. México, D. F.: Fundación SM.

Cruz, F. (3 de febrero de 2018). Iztapalapa, con más violencia; dos de cada 10 delitos. Excélsior. Recuperado de: https://www.excelsior.com.mx/comunidad/2018/02/03/1217883

Esteban-Guitart, M. (2012). La multimetodología autobiográfica extendida (MAE). Una estrategia cualitativa para estudiar la identidad, los fondos de conocimiento y las formas de vida. Revista Electrónica de Metodología Aplicada, 17(2), 51-64.

Esteban-Guitart, M. (2016). Funds of identity. Connecting meaningful learning experiences in and out of school. New York: Cambrigde University Press.

Esteinou, R. (2005). La juventud y los jóvenes como construcción social. En M. Mier y Terán, \& C. Rabel (Coords.), Jóvenes y niños, un enfoque sociodemográfico (pp. 25-35). México, D. F.: Miguel Ángel Porrúa, Instituto de Investigaciones Sociales, Unam.

Feito, L. (2007). Vulnerabilidad. Anales del Sistema Sanitario de Navarra, 30(3), 7-22. Recuperado de: http://scielo.isciii.es/pdf/asisna/v30s3/original1.pdf

Herreros, M. (2012). El uso de los relatos digitales personales como herramienta para pensar el Yo (Self). Digital Education Review, 22, 68-79.

Lambert, J. (2010). Digital Storytelling Cookbook. Berkeley: Center for Digital Storytelling.

Manrique, R. (2015). La cuestión de la inteligencia emocional. Revista de la Asociación Española de Neuropsiquiatría, 35(128), 801-814. doi:10.4321/S0211-57352015000400008

Marchesi, A. (2004). Qué será de nosotros, los malos alumnos. Madrid: Alianza.

Margulis, M. (2001). Juventud: una aproximación conceptual. En S. Donas-Burak (Comp.), Adolescencia y juventud en América Latina (pp. 42-56). Cartago: Libro Universitario Regional.

Ocde. (2016). Los resultados de México en la evaluación 2015 de Pisa. Recuperado de: https://www. 
oecd.org/pisa/PISA-2015-Mexico-ESP.pdf

Ortega, R., Del Rey, R., \& Córdoba, F. (2010). Las aulas de educación secundaria como espacios de convivencia y aprendizaje. En C. Coll (Coord.), Desarrollo, aprendizaje y enseñanza en la educación secundaria (pp.193-208). Barcelona: Graó.

Prensky, M. (2001). Digital natives, digital immigrants part 1. The Horizon, 9(5), 1-6. doi:10.1108/10748120110424816

Reséndiz, A. M. (2016). Identidad, escolaridad y proyecto de vida: factores de resiliencia en jóvenes de Ciudad Juárez, Chihuahua. (Tesis de Maestría en Pedagogía). Universidad Nacional Autónoma de México, México, D. C., México.

Reyes, A. (2016). Adolescencias entre muros. Escuela secundaria y construcción de identidades juveniles. México, D. F.: Flacso.

Ricoeur, P. (2006). La vida: un relato en busca de narrador. Revista Ágora, Papeles de Filosofía, 25(2), 9-22. Recuperado de: https://minerva.usc.es/xmlui/bitstream/handle/10347/1316/Ricoeur.pdf?sequence=1

Rudduck, J, \& Flutter, J. (2007). Cómo mejorar tu centro escolar dando la voz al alumnado. Madrid: Morata.

Sanjurjo, L. (2009). Los dispositivos para la formación en las prácticas profesionales. Rosario: Hommo Sapiens.

Secretaría de Educación Pública (2011). Plan de Estudios. Educación Básica en México. Recuperado de: https://www.gob.mx/sep/documentos/plan-de-estudios-educacion-basica-en-mexico-2011

Urrutia de la Torre, F., \& Martín del Campo, A. (2015). El abandono escolar en el nivel secundaria: un descuido en la agenda educativa actual. Revista Latinoamericana de Estudios Educativos. 45 (1), 63-74. Recuperado de:

http://www.redalyc.org/pdf/270/27035790003.pdf 